\title{
Viewpoint
}

\section{The practice of UK OR practitioners}

Journal of the Operational Research Society (2004) 55, 90. doi:10.1057/palgrave.jors.2601669

Munro and Mingers report on a survey they conducted on the use of 'multimethodology' in practice. ${ }^{1}$ They have produced a large set of data, making clear their approach. They warn that, because of the difficulty of defining a theoretical population of practitioners from both an OR/MS and systems background, the results should be taken as indicative and illuminating rather then as a statistically valid representation. It is tempting to read and then forget such preliminary warnings when absorbing their interesting analysis of the large and rich set of data collected. However, the reservations about the sample are important. I was struck by Figure 3 in the paper, which shows the usage of different methods. The figure indicates that about a third of respondents did not know or had no more than heard of simulation. A similar proportion was barely aware of SSM. A small number had even responded that statistical analysis was unknown to them. Clearly some in the sample, presumably systems people, are unfamiliar with established OR methods, others, presumably OR people, are unfamiliar with the best known systems methodology. This observation led me to return to the nature of the sample. To produce their rich data set and to include as many users of multiple methods as possible, Munro and Mingers have clearly been keen to sweep as many respondents into the sample pot as possible. As they point out, the respondents are predominantly UK OR practitioners but also included are academics, non-UK residents and systems people. Munro and Mingers also report that their data indicate that academics tend to favour the approaches developed at their own institutions (cognitive mapping at Strathclyde, etc); as a result the inclusion of academics makes the interpretation of the data more problematic. Thus quite apart from the usual problems of self-selecting respondents, the data include both very different sorts of practitioners and academics each with a particular research agenda. The results therefore need to be interpreted with care. However, the data provide an opportunity to obtain a somewhat sharper focus on the practice of UK OR practitioners by taking out the remainder of academics, non-UK residents and systems people. One approach would be to separate the first two categories of the survey sample (independent consultants and heads of OR groups) from the second two (attendees at 1997 UK Systems Society Conference and personal contacts/ respondents to adverts). This would presumably be straightforward and provide a pure OR practitioner sample of some interest. The purpose of my viewpoint is therefore to ask Munro and Mingers to respond with some breakdown of their data to highlight the responses of UK OR practitioners. I would find Figure 3 and Tables 3, 5 and 6 of particular interest.

\section{References}

1 Munro I and Mingers J (2002). The use of multimethodology in practice-results of a survey of practitioners. J Opl Res Soc 53: 369-378.

University of Warwick

R Ormerod

\section{Response to Richard Ormerod}

Journal of the Operational Research Society (2004) 55, 90-93. doi:10.1057/palgrave.jors. 2601670

We would like to thank Richard Ormerod for his interest in our survey of the use in practice of multimethodology, ${ }^{1}$ and hope that we can address his concerns in this brief reply. Our survey covers a range of people using multimethodology including splits between OR and systems, and practitioner and academic, as well as some who go across these categories. Ormerod is particularly interested in OR practitioners and we have done our best to split these out from the main sample. A number of important points of difference will be highlighted, which confirm Ormerod's hypothesis that significant differences can be found when looking at the responses from the sample as a whole compared with the responses of the non-academic OR practitioners within that sample. Indeed, his hunch that the use of systems methodologies is largely restricted to the academic respondents of the sample turned out to be correct, at least in terms of designing multimethodologies. On returning to the data it was found that systems methodologies such as SSM, VSM and Cognitive Mapping were hardly used by nonacademic practitioners (NAPs for brevity) in designing multimethodology. 